\title{
Dried human skin fibroblasts as a new substratum for functional culture of hepatic cells
}

\author{
Agnieszka Wencel', Karolina Ewa Zakrzewska', Anna Samluk1, Bartłomiej Henryk Noszczyk², \\ Dorota Genowefa Pijanowska' and Krzysztof Dariusz Pluta ${ }^{凶}$
}

1Department of Hybrid Microbiosystems Engineering, Nalecz Institute of Biocybernetics and Biomedical Engineering, Polish Academy of Sciences, Warsaw, Poland; ${ }^{2}$ Department of Plastic Surgery, Medical Centre of Postgraduate Education, Orlowski Memorial Hospital, Warsaw, Poland

The primary hepatocytes culture is still one of the main challenges in toxicology studies in the drug discovery process, development of in vitro models to study liver function, and cell-based therapies. Isolated hepatocytes display a rapid decline in viability and liver-specific functions including albumin production, conversion of ammonia to urea, and activity of the drug metabolizing enzymes. A number of methods have been developed in order to maintain hepatocytes in their highly differentiated state in vitro. Optimization of culture conditions includes a variety of media formulations and supplements, growth surface coating with the components of extracelIular matrix or with synthetic polymers, three-dimensional growth scaffolds and decellularized tissues, and coculture with other cell types required for the normal cellcell interactions. Here we propose a new substratum for hepatic cells made by drying confluent human skin fibroblasts' culture. This growth surface coating, prepared using maximally simplified procedure, combines the advantages of the use of extracellular matrices and growth factors/cytokines secreted by the feeder layer cells. In comparison to the hepatoma cells grown on a regular tissue culture plastic, cells cultured on the dried fibroblasts were able to synthesize albumin in larger quantities and to form greater number of apical vacuoles. Unlike the coculture with the living feeder layer cells, the number of cells grown on the new substratum was not reduced after fourteen days of culture. This fact could make the dried fibroblasts coating an ideal candidate for the substrate for non-dividing human hepatocytes.

Key words: cocultures, culture substratum, dried fibroblasts, human skin fibroblasts, C3A cells

Received: 23 November, 2016; revised: 15 March, 2017; accepted: 15 March, 2017; available on-line: 09 June, 2017

e-mail: kpluta@ibib.waw.pl

Abbreviations: APOLT, auxilary partial orthotopic liver transplantation; $\mathrm{BAL}$, bioartificial liver devices; $\mathrm{C} 3 \mathrm{~A}$, human hepatoma cell; drHSF, dried human skin fibroblasts; ECM, extracellular matrix; FACS, fluorescence-activated cell sorting; HSF, human skin fibroblasts; MFI, median fluorescence intensity; OLT, orthotopic liver transplantation; PI, propidium iodide; TB, trypan blue

\section{INTRODUCTION}

Liver - the largest organ in the human body volumetrically consists of $80 \%$ of hepatocytes. It is responsible for homeostasis, lipid metabolism, detoxification, production of serum proteins, storage of various substances and many other functions (Evenou et al., 2011). Unfortunately, each year in the United States over 38000 deaths from liver diseases are recorded (Kenneth et al., 2014) and the percentage of such deaths reaches $2.5 \%$ worldwide (Palakkan et al., 2013). Moreover, morbidity and mortality of the liver diseases are continuously increasing (Blachier et al., 2013).

The standard of treatment for patients with liverbased metabolic diseases and end-stage liver diseases is orthotopic liver transplantation (OLT). In less serious cases an auxilary partial orthotopic liver transplantation (APOLT) is also applied (Forbes et al., 2015). However, a shortage of donors, operative complications, and the high cost, reduce the advantages of these procedures (Blachier et al., 2013). According to the Organ Procurement and Transplantation Network annually $10 \%$ of the 15000 people in the United States die while waiting for a liver transplant (OPTN Database 2016). Other types of treatment, such as liver regeneration therapy and liver support devices, require adequate source of functional hepatocytes. There are many alternatives to primary hepatocytes, which may be used depending on the medical applications. Human and porcine hepatocytes, human liver tumor-derived cell lines, human fetal hepatocytes or embryonic/induced pluripotent stem cells are used for the construction of bioartificial liver devices (BAL). In turn, adult stem cells, oval cells or hepatoblasts are applied for cell transplantation. Based on their advantages and disadvantages, certain types of cells are chosen for particular application (Palakkan et al., 2013). Bhatia and coworkers (2014) demonstrated that isolated hepatocytes are the most suitable cells for in vitro applications.

Isolated human hepatocytes, cultured in a monolayer, rapidly dedifferentiate and lose their characteristic functions (Baker et al., 2001; Rowe et al., 2013), such as: albumin secretion, ammonia metabolism, and cell polarity (Sellaro et al., 2010). Due to this fact, a number of attempts to maintain liver-specific properties of hepatocytes in vitro were undertaken. These manipulations involved the addition of soluble growth factors to the medium and providing the isolated hepatocytes with the cell-extracellular matrix (ECM) proteins and cell-cell interactions (Lee \& Lee, 2014). The hepatocyte mitogens (e.g. epidermal growth factor (EGF), transforming growth factor- $\alpha$ (TGF- $\alpha$ ) or hepatocyte growth factor (HGF)) and comitogenic growth factors (e.g. insulin, glucagon, vasopressin, insulin-like growth factor (IGF)-I and IGF-II) were tested as suplements for maintaining a functional rat hepatocytes differentiation profile during culture.

In contrast to the other epithelial cell types, hepatocytes are connected to ECM by the basolateral surfaces, and thus regular polystyrene culture dishes are not 
suitable for hepatocytes culture. The growth surfaces prepared for the long-term hepatocytes cultivation are coated with many different components of the ECM. The most commonly used are: Martigel ${ }^{\circledR}$ - ECM gel from Engelbreth-Holm-Swarm mouse sarcoma, rat tail collagen I, gelled collagen from bovine skin (Panda et al., 2015), and porcine-liver-derived extracellular matrix (PLECM). Additionally, the sandwich cultures, which mimic in vivo situation better than monolayer cell culture systems, were established (Sellaro et al., 2010). For these applications a variety of materials (e.g. collagen, fibronectin, laminin (Sellaro et al., 2010; Yang et al., 2013), vitronectin, and fibrillin (Bartolo et al., 2005; Yang et al., 2013)) was analyzed. Faulk and coworkers (2015) used ECM derived from different species - rat, porcine, and ferret.

The primary human hepatocytes were also cultured in 3D systems prepared from natural and synthetic materials. Many different technologies were developed for hepatocyte spheroid culture, including encapsulations, micropatterned arrays, and rotational cultures (Bhatia et al., 2014). Furthermore, the decellularized tissues or organs (Faulk et al., 2015), especially decellularized wholeliver biological scaffolds, opened a new perspective for hepatocyte culture. The liver scaffold was prepared by the perfusion of the organ with a chemical detergent. Resulting cell-free biological scaffold was suitable for the growth of HepG2 and C3A cells (Cheng et al., 2013) as well as mouse and rat hepatocytes, and human fetal liver or stem cells (Faulk et al., 2015).

The interactions between nonparenchymal and parenchymal cells play crucial role in the liver physiology (Khetani et al., 2004) enabling hepatocytes to maintain their viability and functions for several weeks (Jeong et al., 2016). To support rat hepatocytes functions in culture, influence of nonparenchymal cells, inter alia stellate cells, was tested. Also other types of cocultures were applied: liver-derived cells, including Kupffer cells, sinusoidal endothelial cells, and non-liver-derived cells (e.g. murine embryonic, human lung epithelia, human fibroblasts) (Bhatia et al., 1999).

In this paper, the influence of dried human skin fibroblasts (drHSF), for the first time used as a surface coating, was described. The study was performed to determine whether hepatocytes functions could be maintained without direct heterotypic cell-cell interactions. Since C3A cell line, the clonal derivative of HepG2, is commonly used for hepatotoxicity studies it was used as an experimental model of hepatic cells. The $\mathrm{C} 3 \mathrm{~A}$ is also used in the commercial BAL system by Vital Therapies - ELAD (Extracorporeal Liver Assist Device) (Nelson et al., 2015). We showed that the number of dead cells in population of the hepatic cells (C3A) cultured on the $\mathrm{drHSF}$ is significantly reduced in comparison to the control. Moreover, typical hepatic functions (albumin synthesis, formation of apical vacuoles - functional and structural analogs of bile canaliculi) of $\mathrm{C} 3 \mathrm{~A}$ cells were improved. The positive effect of culturing C3A cells on drHSF was reconfirmed by using different ratios of C3A:HSF/drHSF. The results showed a ratio-dependent improvement of the hepatic cells culture performance. Therefore, we concluded that the contact between hepatocytes and nonparenchymal cells may not be necessary. When the concentration of growth factors preserved in the dried fibroblasts layer is adequate this treatment can maintain hepatic cells functions in vitro. Hence, these results can be helpful in the improvement of the cytotoxic tests' design or BAL constructed using C3A cells. In the future, this approach can also be useful for cultivation of the primary human hepatocytes in simplified culture arrangement.

\section{MATERIALS AND METHODS}

Cells and coculture models. The C3A human hepatoma cell line was purchased from the American Type Culture Collection (ATCC, Manassas, VA, USA) (ATCC No.CRL-10741). The adult human skin fibroblasts (HSF) were isolated from dermis. Skin biopsies were donated by 15 donors operated on for aesthetic breast reduction or mastopexy ( 8 women) and aesthetic abdominoplasty (4 women and 3 men) in the Department of Plastic Surgery, Medical Centre of Postgraduate Education, Orlowski Memorial Hospital in Warsaw. Mean age of the patients was $37.2( \pm 8.82)$ and mean weight was $70.93 \mathrm{~kg}( \pm 13.99)$. The study was approved by the Bioethical Committee of the Medical Centre of Postgraduate Education (decision no. 1/24/01/2007) and performed according to the principles of the Declaration of Helsinki. All the patients provided their written, informed consent. The cells were cultured under standard conditions - in $95 \%$ air $/ 5 \% \mathrm{CO}_{2}$ at $37^{\circ} \mathrm{C}$. The culture media consisted of high glucose DMEM (Dulbecco Modified Eagle Medium, Sigma Aldrich, Poznan, Poland) supplemented with 10\% fetal calf serum (Biological Industries Inc., Beit-Haemek, Israel) and 1\% nonessential amino acids (Biological Industries Inc., BeitHaemek, Israel).

The C3A cells were seeded with the HSF at the same time or on substratum prepared by drying 5 days culture of HSF (drHSF). The drying process was conducted in a laminar flow cabinet at room temperature (RT) for 30 min, and then plates were stored at $4{ }^{\circ} \mathrm{C}$. The cells were seeded in 24-well plates and cultured for 14 days. The C3A cells were seeded at a density of $1.25 \times 10^{4}$ cells per $\mathrm{cm}^{2}$. In the cultures with $\mathrm{HSF} / \mathrm{drHSF}$ cells were seeded in the following C3A to HSF cell number ratios: 3:1 $\left(3.75 \times 10^{3}\right.$ fibroblasts per $\left.\mathrm{cm}^{2}\right), 5: 1\left(2.5 \times 10^{3}\right.$ fibroblasts per $\left.\mathrm{cm}^{2}\right)$, and $10: 1\left(1.25 \times 10^{3}\right.$ fibroblasts per $\left.\mathrm{cm}^{2}\right)$. For control, C3A cells were seeded individually (monoculture). All experiments were triplicated.

Dead/live cells discrimination. The percentage of living cells was measured using trypan blue (TB) exclusion method. Cells were suspended in $0.2 \% \mathrm{~TB}$ and then counted in a hemocytometer to determine the number of cells that had clear cytoplasm (viable cells) and cells that had cytoplasm stained blue (nonviable cells). For flow cytometry (fluorescence-activated cell sorting, FACS) analysis, percentage of dead cells was determined through staining with $5 \mu \mathrm{g} / \mathrm{ml}$ propidium iodide (PI) (Sigma Aldrich, Poznan, Poland).

Apical vacuoles evaluation. The samples of the human hepatoma cells with the HSF/drHSF in appropriate proportions were seeded on glass slides $\left(3.6 \mathrm{~cm}^{2}\right)$. On day 8 samples were fixed with $4 \%$ formaldehyde (ALAB, Warsaw, Poland) at RT for 15 min. Then, glass slides were washed three times with phosphate buffered saline (PBS) and incubated with $0.1 \%$ Triton X-100 for 10 min. The samples were washed and treated with $50 \mu \mathrm{g} /$ $\mathrm{ml}$ phalloidin labeled with tetramethylrhodamine isothiocyanate - TRITC (Sigma Aldrich, Poznan, Poland) for $15 \mathrm{~min}$ at RT. Thereafter the samples were counterstained with 4',6-diamidino-2-phenylindole (DAPI) (Sigma Aldrich, Poznan, Poland). The preparations were embedded in Histofluid (Medlab Products, Raszyn, Poland) and imaged using the Olympus Spinning Disk Confocal Microscope (Olympus, Warsaw, Poland). The 
system consisted of an Olympus IX81 inverted microscope equipped with the Yokogawa spinning disk scanner and EMCCD camera. The apical vacuoles and the nuclei were counted at $200 \times$ magnification in 6 fields of view for each individual culture and at least 13 vacuoles were counted.

Albumin measurement. Culture media samples were collected every second day and stored at $-20^{\circ} \mathrm{C}$ for albumin quantification. The concentration of albumin in each sample was determined by enzyme-linked immunosorbent assay (ELISA) with the quantitation kit (Bethyl Laboratories Inc., Montgomery, TX, USA) following the manufacturer's instructions. The absorbance of samples was read at wavelength of $450 \mathrm{~nm}$ using the Synergy HT microplate reader (Bio-Tek, Winooski, VT, USA). The amount of albumin was expressed in $\mathrm{ng} / \mathrm{ml}$ of the culture medium or in $\mathrm{ng} / 10000$ counted cells.

Flow cytometry analysis. For FACS analysis, cells were harvested from culture plates on day 14. Cells were trypsinized, fixed with $4 \%$ formaldehyde and permeabilized using $0.1 \%$ Triton X 100 solution in PBS. Prepared cell suspensions were incubated with primary antibodies conjugated with fluorescein isothiocyanate (FITC). The goat $\alpha$-human albumin-FITC antibody (Bethyl Laboratories, Inc., Montgomery, TX, USA) was used for FACS. Unstained specimens and cells incubated with appropriate isotype immunoglobulin were used as controls. The stained cells were evaluated by flow cytometry us-
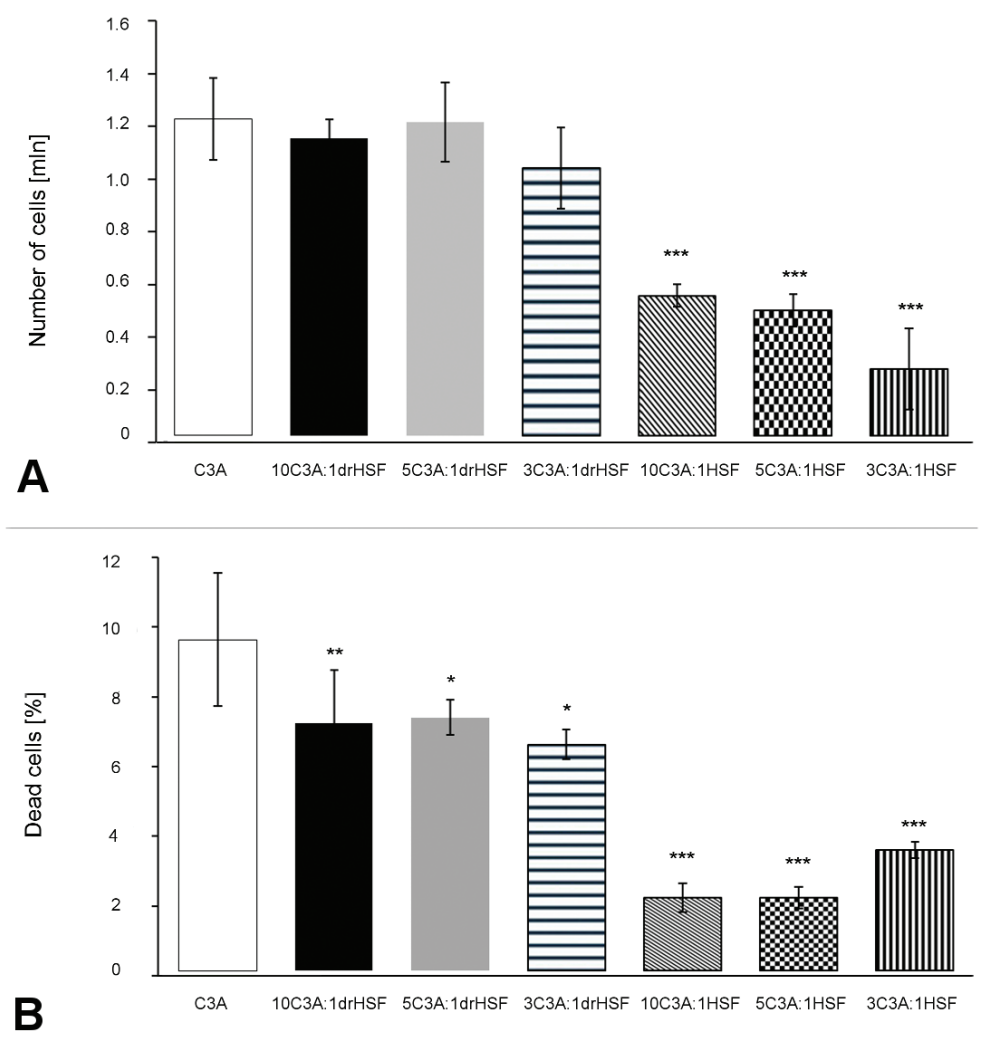

Figure 1. The effects of the cocultures with human skin fibroblasts on number of live and dead C $3 A$ cells

In cocultures, C3A cells and HSF or in cultures of C3A with drHSF the cells were seeded at the following ratios: 10:1, 5:1, and 3:1. For control, C3A cells were seeded individually (monoculture). (A) The number of live cells after 2 weeks of experiment. Live C3A cells were discriminated from the dead cells by trypan blue exclusion method. (B) The percentage of dead C3A cells after 2 weeks of experiment. The percentage of the dead cells was determined through the staining with propidium iodide and flow cytometry (FACS) analysis. The results were analyzed using one-way ANOVA and Duncan's post hoc test. Bars: \pm S.D., $\mathrm{n}=3 . P<0.05\left(^{*}\right), P<0.01$ $(* *), P<0.001(* * *)$. ing FACSCanto II instrument (Becton Dickinson - BD, Warsaw, Poland). FACSDiva software (BD, Warsaw, Pond) was used for data acquisition and analysis.

Statistical analysis. All the data are expressed as STATISTICA 10 software (StatSoft Inc., Tulsa, OK, UA). Data were analyzed using one-way analysis of variance (ANOVA). The differences between the groups were analyzed using Duncan's multiple range test. A $P$-value lower than 0.05 was considered to be statistically

\section{RESULTS}

The number of cells and the percentage of live/ dead cells were quantified on day 14 of the culture. The ture was more than two times lower than in the mono(10 C3A: $1 \mathrm{HSF}=5.3 \times 10^{5}$ cells per well difference could be explained by the presence of ly large growth surface area. Furthermore, there were no statistically significant differences in the number of cells grown on the three versions of the drHSF substratum

Addition of the HSF to the culture or culture on the drHSF significantly decreased number of dead C3A cells (Fig. 1B). In coculture with the HSF there were less than $4 \%$ of dead cells (3.5\% of dead cells at $3: 1$ ratio of $\mathrm{C} 3 \mathrm{~A}$ to HSF, $P<0.001)$, whereas nonviable cells accounted for nearly $10 \%$ of the population in the monoculture. Dried HSF also had a positive impact on C3A cells viability $(7.1 \%$ dead cells at $10: 1 \mathrm{ra}$ tio of $\mathrm{C} 3 \mathrm{~A}$ to $\mathrm{drHSF}, P<0.001 ; 7.3 \%$ at 5:1 ratio, $P<0.01$ and $6.5 \%$ at $3: 1$ ratio, $P<0.01)$ compared to the control $(9.5 \%)$.

Albumin production was confirmed by flow cytometry analysis (Table 1). Growing ratio of the fibroblasts to the $\mathrm{C} 3 \mathrm{~A}$ cells increased albumin levels as assessed by the median fluorescence intensity (MFI). Expression of the albumin in the 3 C3A:1 HSF and the monoculture was 816 and 172, respectively (in relative units of MFI). In the case of the culture carried on the drHSF albumin-related MFI also increased (230 for the culture variant $3 \mathrm{C} 3 \mathrm{~A}: 1 \mathrm{drHSF})$.

To assess the metabolic activity of the human hepatoma cells, albumin secretion by the C3A cells was quantified using the ELISA test. The amount of albumin was expressed in nanograms per $\mathrm{ml}$ of the culture medium or in nanograms per 10000 counted cells. In all variants of the experiment the albumin production showed logarithmic increase with the exception of the control (monoculture), where sudden downgrade from $4358.5 \mathrm{ng} / \mathrm{ml}$ on day 12 to $3181.6 \mathrm{ng} /$ $\mathrm{ml}$ on day 14 was observed (Fig. 2A, B). This could be explained by the fact that C3A cells had already reached confluen- 
Table 1. The results of flow cytometry analysis.

Expression of albumin was determined through the staining of cells with anti-albumin antibody conjugated with FITC and expressed in relative units of median fluorescence intensity (MFI). In cocultures, C3A cells and HSF or in cultures of C $3 \mathrm{~A}$ cells with drHSF the cells were seeded in the following ratios: 10:1, 5:1, and 3:1. For control, C3A cells were seeded individually (monoculture).

\begin{tabular}{lc}
\hline Variants of culture & MFI \\
\hline C3A & 172 \\
\hline 10 C3A:1 drHSF & 125 \\
\hline 5 C3A:1 drHSF & 179 \\
\hline 3 C3A:1 drHSF & 230 \\
\hline 10 C3A:1 HSF & 460 \\
\hline 5 C3A:1 HSF & 751 \\
\hline 3 C3A:1 HSF & 816 \\
\hline
\end{tabular}

cy. The highest amounts of the albumin were detected in the medium harvested from the $\mathrm{C} 3 \mathrm{~A} / \mathrm{drHSF}$ cultures (Fig. 2A). The lowered production of the protein on day 14 in the 3 C3A:1 HSF (2710.9 ng/ml) (Fig. 2B) in comparison to the $3 \mathrm{C} 3 \mathrm{~A}: 1 \mathrm{drHSF}(5908.3 \mathrm{ng} / \mathrm{ml})$ (Fig. 2A) was presumably caused by the smaller number of $\mathrm{C} 3 \mathrm{~A}$ cells in the coculture with the living fibroblasts (Fig. 1A). The proliferating fibroblasts held down C3A cells growth, thereby decreasing the total amount of secreted protein. Minor differences between the variants
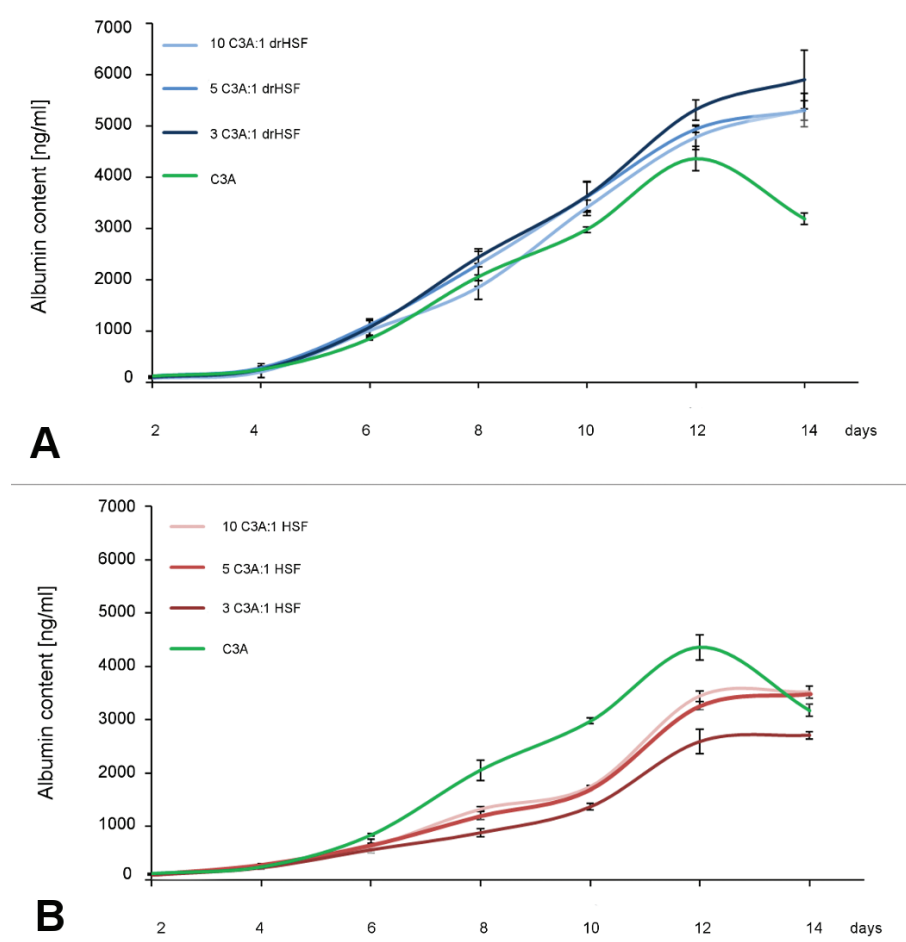

Figure 2. Production of albumin by C3A cells in the particular days of the

Albumin secretion by the cultured hepatic cells was measured in the medium using ELISA assay. Samples of the medium were taken every second day for 2 weeks of the culture. In cultures of C3A cells with drHSF or cocultures with HSF the cells were seeded at the following ratios: 10:1, 5:1, and 3:1. For control, C3A cells were seeded individually (monoculture). (A) Production of albumin in the particular days of the culture of C3A cells with drHSF. (B) Production of albumin in the particular days of the culture of C3A cells with HSF. The amount of albumin was expressed in $\mathrm{ng} / \mathrm{ml}$ of the culture medium. Bars: \pm S.D., $n=3$. were observed. When the amount of albumin was normalized to a final number of cells (Fig. 3), higher levels of the albumin were present in the culture variants containing increasing number of the HSF. Production of the albumin was four times higher in the coculture 3 C3A:1 HSF (107.0 ng \pm 2.6 per 10000 cells) compared to monoculture (26.5 ng \pm 0.9 per 10000 cells, $P<0.001)$. The lowest production of the albumin was observed in the $5 \mathrm{C} 3 \mathrm{~A}: 1 \mathrm{drHSF}$ (44.7 ng \pm 1.6 per 10000 cells), yet the difference between this culture variant and the control was still statistically significant $(P<0.001)$.

After 8 days of the culture apical vacuoles formation (Fig. 4A, B) was determined. Quantitative analysis of the apical vacuoles is presented in Fig. 4C as the number of the vacuoles divided by the number of nuclei and expressed as the percentage. The mean number of these structures in $\mathrm{C} 3 \mathrm{~A}$ cells with the $\mathrm{HSF} / \mathrm{drHSF}$ was about $20 \%$ higher than in the control, but no differences between various ratios of $\mathrm{C} 3 \mathrm{~A}$ cells and the fibroblasts in coculture were observed. The percentage of the apical vacuoles ranged from $28.3 \% \pm 2.3$ in the $3 \mathrm{C} 3 \mathrm{~A}: 1 \mathrm{drHSF}$ to $29.5 \% \pm 0.9$ in the $10 \mathrm{C} 3 \mathrm{~A}: 1 \mathrm{HFS}(23.2 \% \pm 3.3$ in the control monoculture).

\section{DISCUSSION}

The objective of this study was to develop the potentially usefull hepatic cells attachment and growth substratum as a new tool for in vitro applications. We proposed growth surface coating prepared by drying 5 days culture of human skin fibroblasts - drHSF. Fibroblasts appear to be relevant for hepatocytes culture because they excrete diverse factors, such as ECM proteins (e.g. collagen I, decorin) and cytokines (e.g. interleukin-1, interleukin-6, vascular endothelial growth factor (VEGF)) (Jeong et al., 2016). However, it seems that heterotypic cell-cell interactions are more important for hepatic cells metabolic activity than the presence of growth factors in the culture medium. Hui \& Bhatia (2007) demonstrated that matrix deposition and direct cell-cell interaction maintain hepatocyte functions in cocultures, whereas soluble factors are ineffective. Our preliminary data showed that the new type of growth surface coating might combine the effect of the cell-ECM contacts with the accessibility of growth factors. In turn, the lack of living feeder layer cells allowed us to avoid problems with overgrowing the coculture with the fibroblasts, which leads to the reduced number of hepatic cells.

A growing body of research in the field of human hepatocytes primary cultures, conducted over the last two decades, showed significant advances, yet further studies are required to obtain satisfying results. Bhatia and coworkers (1999) presented the summary, demonstrating positive impact of both liver-derived cells (including: liver biliary epithelial cells, stellate cells, sinusoidal endothelial cells) and numerous non-liver-derived cells (e.g. embryonic fibroblasts) on rat hepatocytes' phenotype. In 2008 the use of murine 3T3-J2 fibroblasts as a new technique for primary adult rat hepatocytes expansion in vitro was described. To assess the effect of coculture three types of fibroblasts were used: 3T3-J2, NIH-3T3, and mouse em- 


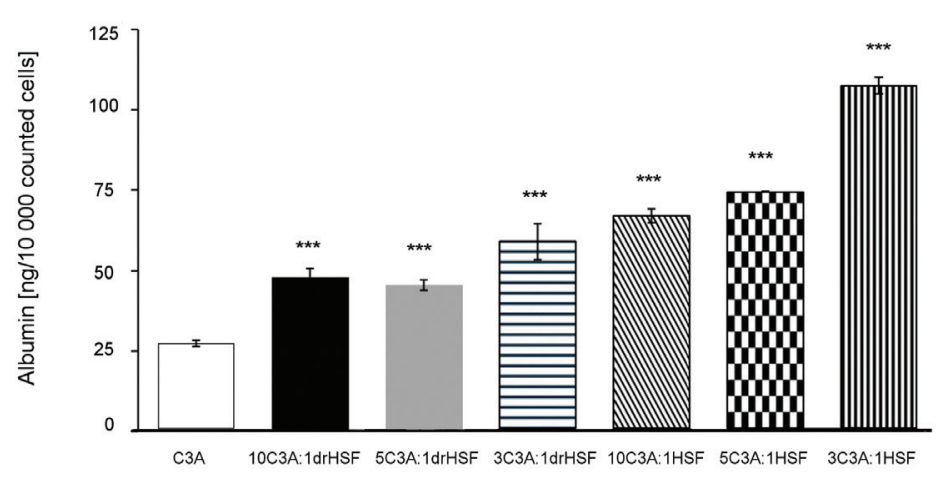

Figure 3. Albumin production per 10 thousand counted cells on the culture day 14

Albumin secretion by the cultured hepatic cells on day 14 was measured using ELISA assay and presented in ng of the protein calculated per $1 \times 10^{4}$ counted cells. Samples of the medium were taken in the last 2 days of the culture. In cocultures, C $3 A$ cells and HSF or in cultures of C3A cells with drHSF the cells were seeded at the following ratios: 10:1, 5:1, and 3:1. For control, C3A cells were seeded individually (monoculture). Results were analyzed using one-way ANOVA and Duncan's post hoc test. Bars: \pm S.D., $\mathrm{n}=3 . P<0.001$ ( $\left.^{* * *}\right)$.
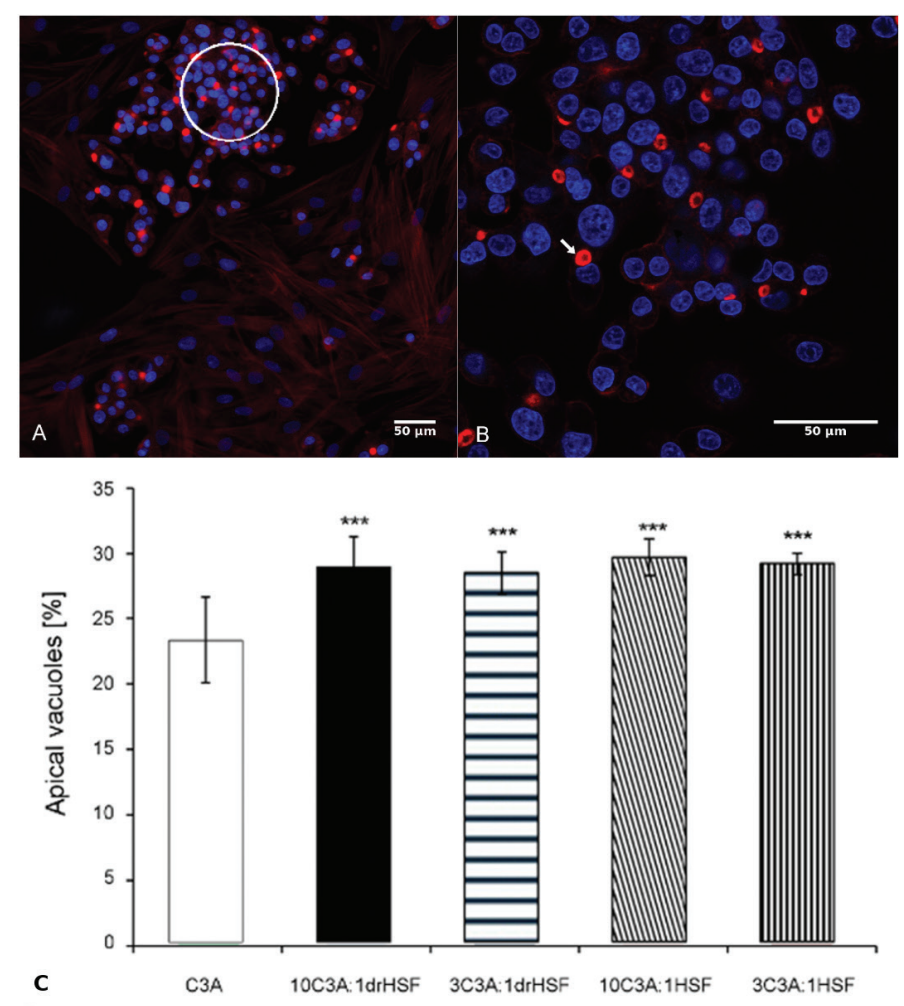

Figure 4. Apical vacuoles formation after 8 days of the culture of C3A cells with HSF and drHSF

Cells on day 8 were fixed and permeabilized using formaldehyde and Triton X-100, respectively. The fixed samples were stained with TRITC-conjugated phalloidin. This probe was used for labeling of the actin filaments (red). The nuclei were counter-stained with DAPI (blue). (A) Apical vacuoles formation after 8 days of the culture of C3A cells with HSF (3 C3A:1 HSF). "Island" of C3A cells (white circle), surrounded by the HSF. The image was taken at $10 \times$ magnification. (B) area in white circle from Figure $(\mathbf{A})$ in $60 \times$ magnification, white arrow points at the apical vacuole. Scale bars: $50 \mu \mathrm{m}$. (C) The percentage of the apical vacuoles in the 8 day culture of C3A cells with HSF and drHSF. In the cocultures, C3A cells and HSF or in cultures of $\mathrm{C} 3 \mathrm{~A}$ cells with drHSF the cells were seeded at the following ratios: 10:1 and 3:1. For control, C3A cells were seeded individually (monoculture). The results were analyzed using one-way ANOVA and Duncan's post hoc test and presented as a percentage of the apical vacuoles in relation to the number of C3A cells. The apical vacuoles and the nuclei were counted at 200× magnification in 6 fields of view for each individual culture; at least 13 vacuoles were counted. Bars: \pm S.D. $P<0.001$ $(* * *)$. bryonic fibroblasts (MEF). The best results, in all measured parameters - colony size, albumin secretion, urea synthesis, were obtained when a low density culture of growth-arrested 3T3-J2 fibroblasts was used as a feeder layer. This feeder layer, when compared to other types of cells, induced higher hepatocytes proliferation and more pronounced liver-specific functions (Cho et al., 2008). Khetani and coworkers (2004) tested induction of liver-specific functions in rat hepatocytes upon coculture with the same types of murine fibroblasts. The inductive capacity of these types of fibroblasts was then ranked in following order: 3T3-J2 $>\mathrm{NIH}$ 3T3 $>$ MEF. The fibroblasts isolated from different tissues are heterogenous populations, which contain cells of various properties. Cho and coworkers (2008) presumed that the major reason for different proliferative responses of hepatic cells may be characteristic for the coculture cell type - variations in cell signaling, growth factor release, ECM deposition, and proteins production. The application of other types of fibroblasts in cocultures could change the metabolic functions of the tested hepatic cells. Based on these results an examination of various types of dried fibroblasts would be desired.

To our best knowledge this is the first article that describes the properties of dried human skin fibroblasts as a substratum in hepatic cells culture, instead of using dividing feeder layer cells or cells blocked in the metaphase. The substratum prepared by drying the human skin fibroblasts did not change the number of living cells (Fig. 1A), whereas coculture with living HSF significantly reduced the growth rate of $\mathrm{C} 3 \mathrm{~A}$ cells $(P<0.001)$. In turn, drHSF did not affect the number of dead cells $(P<0.01)$ in versions 5 C3A:1 drHSF and 3 C3A:1 drHSF (Fig. 1B), while all versions of the experiments with the HSF significantly decreased it $(P<0.001)$. It was remarkable that apical vacuoles formation was also enhanced in both drHSF and HSF $(P<0.001)$ cultures (Fig. 4C). This shows that the drHSF coating allows hepatocytes to maintain their polarity, and hence, hepatic transport activity might be retained.

Albumin production in monoculture control was severely lowered on day 12 , whereas it was on a steady level after 12 days of the culture in C3A cells with HSF (Fig. 2B). The continous growth of albumin secretion in $\mathrm{C} 3 \mathrm{~A}$ cells cultured on drHSF during 14 days of experiment (Fig. 2A) is particularly important for a long-term culture of liver cells. The reason for this phenomenon may be the contact inhibition in monoculture. The dried substratum presumably eliminates this effect more effectively than living fibroblasts. Additionally, albumin production in all versions of the experiment 
with the drHSF was higher, whereas with the HSF it was lower than in the monoculture (Fig. 2A, B). When the results were calculated in nanograms per 10000 counted cells (Fig. 3), total albumin production on day 14 in the HSF as well as drHSF cultures was statistically higher than in the monoculture $(P<0.001)$. The hepatic cells in the coculture with the HSF at ratios 3:1 produced the highest amount of albumin per cell. This type of culture provides a range of interactions including cytokines production, ECM component production as well as cell-cell contacts. More noticeable results were observed when higher ratios of the fibroblasts to $\mathrm{C} 3 \mathrm{~A}$ cells were used - in both HSF and drHSF cultures. Changing ratios of cells had an impact on the intensity of the homoand heterotypic interactions in the coculture with the $\mathrm{HSF}$ and homotypic interactions on the drHSF, where the C3A did not form island-like aggregates. Previously, Samluk and coworkers (2013) examined the role of homo- and heterotypic interactions in the C3A and HSF cocultures. These results showed that the smaller "islands" formed by hepatic cells surrounded by fibroblasts, the greater heterotypic interactions.

Flow cytometry analysis (Table 1) confirmed ELISA results that along with the increasing ratio of the HSF/ $\mathrm{drHSF}$ to the $\mathrm{C} 3 \mathrm{~A}$, the level of albumin accumulated in the hepatoma cells rose. However, this increase was less prominent in the C3A cells grown on the drHSF. This may be due to the fact that ELISA quantifies the amount of albumin accumulated in the medium for few days of culture, whereas flow cytometry analysis shows only the current albumin level inside the cells. Thus, in this case, ELISA can be a more sensitive method to measure albumin secretion.

One can assume that in the drHSF some levels of growth factors particles were preserved in the surface coating even when the feeder layer cells had been dried. Soto-Gutierrez and coworkers (2011) showed that liver decellularization protocol did not change the hepatic matrix ultrastructure. Moreover, nearly $50 \%$ of the growth factors particles: HGF and basic fibroblast growth factor (bFGF) were retained in the ECM after detergent treatment of the organ. It would be interesting for future investigations to identify fibrolasts' mitogens and other factors embedded in the drHSF surface coating.

Typically, various components of the ECM are applied as an attachment and growth substratum for hepatic cells. This approach, commonly used for improvement of the living cells adhesion, was tested by Shoji and coworkers (2000) on the human hepatoma HepG2 cells. The authors investigated three kinds of matrix proteins and their combinations (collagen, pronectin, fibronectin). Nevertheless, they focused only on the one of the experimental aspects - cells adherence. This feature is important, however, more interesting are other hepatocyte functions such as albumin production and apical vacuoles formation. We studied drHSF coating because, presumably, large portions of the ECM proteins as well as growth factors are retained in the layer of the dried fibroblasts. In the article published by Kono \& Roberts (1996) the collagen alone had no statistically significant influence on the albumin production in the HepG2 cells. The human hepatoma cells used for our experiments readily attached to the tissue culture polystyrene surface, and thus the proposed substratum was not intended to enhance the adhesion but to improved the metabolic functions of the liver cells. Gatmaitan and coworkers (1983) tested the synergistic interactions of hormones and collagenous substrata, because the rat hepatoma cell line could not survive on the tissue culture plastics in a serum-free medium. They focused on the growth parameters (doubling time, saturation density, attachment, and plating efficiency) and enzymatic assays (UDPGT, GST, and TAT). The collagenous substrata could at least support the survival of the rat hepatoma in the serum-free medium lacking growth factors. Authors showed that the growth and/or differentiation of the cells is affected by synergistic interplay between hormones and the substrata. Based on the abovementioned studies we concluded that substratum proposed by us is better than ECM components used on their own because it consists of a mixture of both growth factors and structural proteins.

Last but not least, the dried substratum did not stake out a place for the hepatic cells growth, which was observed in the HSF coculture, where number of living cells on day 14 had decreased significantly in all versions of the culture. These data highlight the capacity of the drHSF to sustain metabolic functions of the C3A via the direct interactions with this substratum. Most interestingly, the best effect was found in the $3 \mathrm{C} 3 \mathrm{~A}: 1 \mathrm{drHSF}$ cell number ratio, therefore we plan to perform next experiments with cultures using other cell proportions, e.g. 2 C3A:1 drHSF or $1 \mathrm{C} 3 \mathrm{~A}: 1 \mathrm{drHSF}$ and elucidate the role of the drHSF in hepatic cells culture. We propose the use of drHSF coating as a simple and cost-effective attachment and growth substratum for human hepatocytes primary cultures. Additional advantage of the proposed substratum over the use of feeder layer cells is that coated plates can be prepared before use and kept refrigerated.

In conclusion, an inexpensive and efficient method for supporting functional hepatic cells for in vitro studies was described. The hepatic cells expressed hepatocytespecific marker protein - albumin and showed normal morphology when grown on the drHSF surface coating (not shown). Moreover, they formed characteristic apical vacuoles, structures typical for polarized hepatic cells. Human skin fibroblasts as a feeder layer were proved to be an effective matrix that supported functional hepatocytes culture for more than two weeks. The major difference between this work and previous studies is the use of the dried human fibroblasts as a substratum. Despite the fact that living feeder layer cells effectively enhanced hepatocyte-specific functions of the C3A cells, cultures performed on the dried fibroblasts substratum delivered the highest amounts of albumin. It is of particular importance in such applications as BAL where the productiveness of a cartridge housing liver cells is more important than the metabolic capacity of an individual cell. Using this natural substratum for the first time, we clearly demonstrated that factors embedded in dried feeder layer can support hepatic functions and viability without direct cell-cell contacts and without growth surface competition between hepatic cells and fibroblasts. It is especially important in the case of isolated human hepatocytes, which are unable to proliferate. The advantage of proposed substratum is that growth factors remain in close contact with the cells instead of being dissolved in the medium. The role of the dried fibroblasts in the hepatic cells metabolism is to be determined. Further research will be focused on the identification of the factors responsible for improvement of the hepatic cells culture parameters. Moreover, this model of the liver cells culture could be applied in BALs and in in vitro cytotoxicity tests.

\section{Author Contributions}

Conceived and designed the experiments: KDP, KEZ. Performed the experiments: AW, KEZ. Analyzed the 
data: AW, KEZ, AS, KDP. Contributed reagents/materials/analysis tools: DGP, BHN. Wrote the paper: AW, KEZ, DGP, KDP. All authors reviewed the manuscript.

\section{REFERENCES}

Baker TK, Carfagna MA, Gao H, Dow ER, Li Q, Searfoss GH et al., (2001) Temporal gene expression analysis of monolayer cultured rat hepatocytes. Chem Res Toxicol 14: 1218-1231

Bartolo L De, Morelli S, Lopez LC, Giorno L, Campana C, Salerno S, et al., (2005) Biotransformation and liver-specific functions of human hepatocytes in culture on RGD-immobilized plasma-processed membranes. Biomaterials 26: 44324441. http://doi.org/10.1016/j.biomaterials.2004.11.009

Bhatia SN, Balis UJ, Yarmush ML, Toner M (1999) Effect of cell - cell interactions in preservation of cellular phenotype: cocultivation of hepatocytes and nonparenchymal cells. FASEB J 13: 1883-1900

Bhatia SN, Underhill GH, Zaret KS, Fox IJ (2014) Cell and tissue engineering for liver disease. Sci Transl Med 6: $245 \mathrm{st} 2$. http://doi.org/10.1126/scitranslmed.3005975

Blachier M, Leleu H, Peck-Radosavljevic M, Valla D, Roudot-Thoraval F (2013) Special Review The burden of liver disease in Europe: A review of available epidemiological data. I Hepatol 58: 593-608. http://doi.org/10.1016/j. jhep.2012.12.005

Cheng Y, Wang Y, Kang YZ, Hu PY, Gao Y, Pan MX (2013) In vitro culture of tumour-derived hepatocytes in decellularised whole-liver biological scaffolds. Digestion 87: 189-195. http://doi.org/10.1159\%000349949

Cho CH, Berthiaume F, Tilles AW, Yarmush ML (2008) A new technique for primary hepatocyte expansion in vitro. Biotechnol Bioeng 101: 345-356. http://doi.org/10.1002/bit.21911

Evenou F, Hamon M, Fujii T, Takeuchi S, Sakai Y (2011) Gas-permeable membranes and co-culture with fibroblasts enable high-density hepatocyte culture as multilayered liver tissues. Biotech Progr 27: 1146-1153. http://doi.org/10.1002/ btpr.626

Faulk DM, Wildemann JD, Badylak SF (2015) Decellularization and cell seeding of whole liver biologic scaffolds composed of extracellular matrix. J Clin Exp Hepatol 5: 69-80. http:// doi.org/10.1016/j.jceh.2014.03.043

Forbes SJ, Gupta S, Dhawan A (2015) Cell therapy for liver disease: From liver transplantation to cell factory. I Hepatol 62 (Suppl 1): 157-169. http://doi.org/10.1016/j. jhep.2015.02.040

Gatmaitan Z, Jefferson DM, Ruiz-Opazo N, Biempica L, Arias IM, Dudas G et al., (1983) Regulation of growth and differentiation of a rat hepatoma cell line by the synergistic interactions of hormones and collagenous substrata. I Cell Biol 97: 1179-1190

Hui EE, Bhatia SN (2007) Micromechanical control of cell cell interactions. Proc Natl Acad Sci USA 104: 5722-5726. http://doi.org/10.1073/pnas.0608660104

Jeong D, Han C, Kang I, Park HT, Kim J et al., (2016) Effect of concentrated fibroblast - conditioned media on in vitro maintenance of rat primary hepatocyte. PLoS ONE 11: 1-14. http://doi.org/10.1371/journal.pone.0148846

Kenneth D et al., Deaths: Final Data for 2014. National Vital Statistics Reports. 65(4) [cited 2016 Sep 22]. Available from: http://www.cdc.gov/nchs/data/nvsr/nvsr65/nvsr65_04.pdf

Khetani SR, Szulgit G, Rio JA Del, Barlow C, Bhatia SN (2004) Exploring interactions between rat hepatocytes and nonparenchymal cells using gene expression profiling. Hepatology 40: 545-554. http://do1.org/10.1002/hep.20351

Kono Y, Roberts EA (1996) Modulation of the expression of liver-specific functions in novel human hepatocyte lines cultured in a collagen gel sandwich configuration. Biochem Biophys Res Commun 220: 628-632. http://doi.org/10.1006/ bbrc.1996.0454

Lee DH, Lee KW (2014) Hepatocyte isolation, culture, and its clinical applications. Hanyang Med Rev 34: 165-172. http:// doi.org/10.7599/hmr.2014.34.4.165

Nelson LJ, Navarro M, Treskes P, Samuel K, Tura-Ceide O, Morley SD, et al., (2015) Acetaminophen cytotoxicity is ameliorated in a human liver organotypic co-culture model. Sci Rep 5: 17455. http://doi.org/10.1038/srep17455

OPTN Database. National Data. [cited 2016 Sep 22]. Available from: http://optn.transplant.hrsa.gov/data/view-data-reports/national-data/

Palakkan AA, Hay DC, Pr AK, Tv K, Ross JA (2013) Liver tissue engineering and cell sources: Issues and challenges. Liver Int 33: 666-676. http://doi.org/10.1111/liv.12134

Panda S, Bisht S, Malakar D, Mohanty AK, Kaushik JK (2015) In vitro culture of functionally active buffalo hepatocytes Isolated by Using a Simplified Manual Perfusion Method. PLOS ONE 10: 1-17. http://doi.org/10.1371/journal. pone.0118841

Rowe C, Gerrard DT, Jenkins R, Berry A, Durkin K, Sundstrom L, et al., (2013) Proteome-wide analyses of human hepatocytes during differentiation and dedifferentiation. Hepatology 58: 799-809. http://doi.org/10.1002/hep.26414

Samluk A, Zakrzewska KE, Pluta KD (2013) Generation of fluorescently labeled cell lines, C3A hepatoma cells, and human adult skin fibroblasts to study coculture models. Artif Organs 37: E123-E130. http://doi.org/10.1111/aor.12064

Sellaro TL, Ranade A, Faulk DM, Mccabe GP, et al., (2010) Maintenance of human hepatocyte function in vitro by liverderived extracellular matrix gels. Tissue Eng Part A 16: 10751082. http://doi.org/10.1089/ten.tea.2008.0587

Shoji R, Sakai Y, Sakoda A, Suzuki M (2000) Preservation of microplate-attached human hepatoma cells and their use in cytotoxicity tests. Cytotechnology 32: 147-155. http://doi. org/10.1023/A:1008124228377

Soto-Gutierrez A, Zhang L, Medberry C, Fukumitsu K, Faulk D, Jiang H, et al., (2011) A whole-organ regenerative medicine approach for liver replacement. Tissue Eng Part C Methods 17: 677-686. http://doi.org/10.1089/ten.TEC.2010.0698

Yang Y, Li J, Pan X, Zhou P, Yu X, Cao H, et al., (2013) Co-culture with mesenchymal stem cells enhances metabolic functions of liver cells in bioartificial liver system. Biotechnol Bioeng 110: 958-968. http://doi.org/10.1002/bit.24752 